\title{
Evaluation of Athens International Airport Service Quality through the Study of User-Generated Content
}

\author{
Georgios Iliopoulos ${ }^{1}$ and Dimitrios Kafetzopoulos ${ }^{2}$ \\ ${ }^{1}$ Hellenic Open University \\ ${ }^{2}$ University of Macedonia
}

\begin{abstract}
The interest in measuring the elements of airport service quality has been increasing over the last 25 years, keeping pace with the increased availability of commercial non-aeronautical services in airport terminals and the privatization of airport operators worldwide. The Airport Service Quality programme of Airports Council International (ACI/ASQ) has been the basis for many recent surveys on airport quality issues. However, the results of ACI/ASQ are communicated only to the ACI member airports, while this programme includes only those airports that choose to participate. The rapidly increasing mobile internet connectivity over the previous decade has allowed every user of an airport's services to directly post comments on the services provided, even in real time. A number of research articles in recent years have explored whether this usergenerated content can be used to evaluate the quality of an airport's services, often by utilising sentiment analysis tools which are widely available on the internet. As part of this study we investigate which service areas of Athens International Airport are the ones that customers value most according to the comments available on the Google and Twitter platforms, which areas collect the most positive and which the most negative comments, as well as if and how these results differ, depending on the original language of the comment (Greek and non-Greek). Finally, we evaluate two sentiment analysis tools (AFINN and Twinword) with regard to their ability to provide us with valid results on the assessment of user-generated content.
\end{abstract}

Keywords: AFINN; Google; sentiment analysis; Twinword; Twitter 